\title{
Impacto dos sintomas de ansiedade e depressão na autoeficácia percebida em estudantes de enfermagem
}

Impact of anxiety and depression symptoms on perceived self-efficacy in nursing students

Impacto de los síntomas de ansiedad y depresión en la autoeficacia percibida de estudiantes de enfermería

Heloísa Eleotério de Melo ${ }^{1}$ id https://orcid.org/0000-0001-7731-361x

Patricia Fernandes Garcia Severian ${ }^{1}$ is https://orcid.org/0000-0002-5605-2577

Letícia Palota Eid² id https://orcid.org/0000-0002-9413-563X

Marise Ramos de Souza ${ }^{2}$ id https://orcid.org/0000-0002-5361-7819

Carlos Alberto da Cruz Sequeira ${ }^{3}$ io https://orcid.org/0000-0002-5620-3478

Maria da Graça Girade Souza ${ }^{1}$ io https://orcid.org/0000-0003-1754-818x

Daniele Alcalá Pompeo ${ }^{1}$ io https://orcid.org/0000-0003-2671-2586

Como citar:

Melo HE, Severian PF, Eid LP, Souza MR, Sequeira

$\mathrm{CA}$, Souza MG, et al. impacto dos sintomas de

ansiedade e depressão na autoeficácia percebida em estudantes de enfermagem. Acta Paul Enferm. 2021;34:eAPE01113.

DOI

http://dx.doi.org/10.37689/actaape/2021A001113

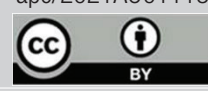

Descritores

Autoeficácia; Autoimagem; Ansiedade; Depressão; Estudantes de enfermagem

Keywords

Self efficacy; Self concept; Anxiety; Depression;

Students, nursing

Descriptores

Autoeficacia; Autoimagem; Ansiedad; Depresión; Estudiantes de enfermería

\section{Submetido \\ 13 de Maio de 2020 \\ Aceito \\ 26 de Agosto de 2020}

Autor correspondente

Daniele Alcalá Pompeo

E-mail: daniele.pompeo@famerp.br

\section{Resumo}

Objetivo: Identificar a relação da autoeficácia percebida com a autoestima e a presença de sintomas de ansiedade e depressão de estudantes no início do curso de graduação em Enfermagem.

Métodos: Estudo transversal, desenvolvido em duas Instituições de Ensino Superior públicas. A amostra foi constituída por 82 participantes. Utilizaram-se três instrumentos validados para mensuração das variáveis: Escala de Autoestima Rosenberg, Escala de Autoeficácia Geral e Percebida e Escala Hospitalar de Ansiedade e Depressão.

Resultados: A maioria dos estudantes apresentou sintomas ansiosos $(73,2 \%)$ e níveis moderados de autoestima $(89,0 \%)$ e de percepção de autoeficácia. Os universitários com sintomas de ansiedade e depressão apresentaram escores de autoeficácia percebida inferiores àqueles sem ansiedade e sem depressão $(p<0,001)$.

Conclusão: A presença de ansiedade e depressão compromete a percepção de autoeficácia de graduandos de enfermagem. Esses resultados adequam-se como um estímulo para a implementação de programas de ajuda em saúde mental nas universidades, que, em médio e longo prazos, poderão propiciar enfermeiros mais saudáveis, satisfeitos e que proporcionarão a seus clientes uma assistência competente e segura.

\section{Abstract}

Objective: To identify the relationship between perceived self-efficacy and self-esteem and the presence of anxiety and depression symptoms in students at the beginning of the undergraduate nursing course.

Methods: Cross-sectional study developed in two public Higher Education Institutions. The sample consisted of 82 participants. Three validated instruments were used to measure the variables: Rosenberg Self-Esteem Scale, General Self-Efficacy Scale and Hospital Anxiety and Depression Scale.

Results: Most students showed anxious symptoms (73.2\%) and moderate levels of self-esteem (89.0\%) and perceived self-efficacy. College students with anxiety and depression symptoms had lower scores of perceived self-efficacy than those without anxiety and depression $(p<0.001)$.

Conclusion: The presence of anxiety and depression compromises undergraduate nursing students' perceived self-efficacy. These results are adequate stimulus for the implementation of mental health care programs at universities, which, in the medium and long term, may provide healthier, more satisfied nurses that will offer competent and safe care to their clients. 


\section{Resumen}

Objetivo: Identificar la relación de la autoeficacia percibida con la autoestima y la presencia de síntomas de ansiedad y depresión de estudiantes al inicio de la carrera de grado de Enfermería.

Métodos: Estudio transversal, llevado a cabo en dos instituciones públicas de educación superior. La muestra estuvo compuesta por 82 participantes. Se utilizaron tres instrumentos validados para la medición de las variables: Escala de Autoestima Rosenberg, Escala de Autoeficacia General y Percibida y Escala Hospitalaria de Ansiedad y Depresión.

Resultados: La mayoría de los estudiantes presentó síntomas ansiosos (73,2 \%) y niveles moderados de autoestima (89,0 \%) y de percepción de autoeficacia. Los universitarios con síntomas de ansiedad y depresión presentaron una puntuación de autoeficacia percibida inferior a aquellos sin ansiedad ni depresión $(p<0,001)$.

Conclusión: La presencia de ansiedad y depresión compromete la percepción de autoeficacia de estudiantes universitarios de enfermería. Estos resultados se presentan como un estímulo para la implementación de programas de ayuda en salud mental en las universidades que, en el medio y largo plazo, podrán proporcionar enfermeros más saludables, satisfechos y que ofrecerán a sus clientes una atención competente y segura.

\section{Introdução}

Estudantes universitários vivenciam situações estressantes e obstáculos pessoais e profissionais que necessitam de estratégias de enfrentamento saudáveis para superação positiva destes fatores negativos. Aqueles que cursam o primeiro ano da universidade estão mais expostos a conflitos, principalmente, relacionados ao gerenciamento do tempo, necessário para organização de sua nova rotina acadêmica e pessoal. Além disso, grande parte desses graduandos está longe de casa pela primeira vez, deparando-se com uma rede de apoio familiar e financeira limitada, ao mesmo tempo em que precisa de recursos emocionais para adaptar-se, com sucesso, a novos contextos sociais e acadêmicos. ${ }^{(1-3)}$

A exigência que se impóe nesse período de transição do ensino médio para o superior, somada a novas atividades e competências necessárias para formação do enfermeiro, podem gerar instabilidade emocional e transtornos psicológicos. Todo este contexto novo propicia condiçôes de vulnerabilidade para o desenvolvimento de estresse, ${ }^{(1)}$ baixa autoestima, ${ }^{(4)}$ transtornos de ansiedade e depressão, ${ }^{(2,5)}$ risco de suicídio e suicídio ${ }^{(6)}$ em estudantes de enfermagem.

Enfrentar os desafios impostos nessa etapa exige autoavaliação e mobilização contínua de recursos internos e externos para lidar com o novo viver. Assim, ter uma atitude positiva em relação a si mesmo pode auxiliar o indivíduo a encarar os infortúnios da vida acadêmica e, posteriormente, a sua vida profissional. $^{(2,3)}$

Nessa perspectiva, a autoeficácia aparece como um importante constructo associado à saúde mental de jovens universitários. Autoeficácia é a crença que a pessoa possui sobre suas capacidades de executar e organizar tarefas com efeito desejado. Essas crenças na eficácia pessoal podem afetar as escolhas de vida, o nível de motivação, a resiliência perante adversidades e a vulnerabilidade ao estresse, ansiedade e depressão. ${ }^{(7)}$

Possuir níveis elevados de autoeficácia é vital para estudantes de enfermagem e enfermeiros. Dentro do contexto acadêmico, se os alunos acreditam em suas capacidades, eles usam todos os seus recursos e se esforçam ao máximo, em diferentes situaçôes, para o alcance de objetivos. Ao contrário, alunos e enfermeiros com baixa autoeficácia não tomariam as ações necessárias para seus pacientes, desenvolveriam uma baixa autoestima clínica e, naturalmente, não iniciariam tarefas sem a certeza de suas habilidades, na busca de se blindar contra erros, ao mesmo tempo, em que privaria seus clientes de uma assistência integral, de qualidade e eficaz. ${ }^{\left({ }^{(8)}\right.}$

A literatura nacional e internacional que versa sobre a autoestima, ${ }^{(1,4,6,9)}$ ansiedade e depressão ${ }^{(2-5)}$ em estudantes de enfermagem é relativamente sólida, apesar de ainda apontar lacunas no comportamento dessas variáveis. Estudos clínicos direcionados a melhora da saúde mental na população universitária têm apresentado resultados favoráveis, entretanto são centrados no gerenciamento da ansiedade, depressão e estresse. ${ }^{(10)}$

Esse cenário pode advir da escassez de evidências consistentes da relação da autoeficácia com outros fatores que interferem no bem-estar mental. Desta forma, a valorização dos aspectos positivos do indivíduo, como a eficácia pessoal, constitui-se um recurso determinante para a promoção da saúde 
mental, dando ênfase à edificação das habilidades socioemocionais do indivíduo, em detrimento do modelo centrado na doença. Portanto, os resultados dessa pesquisa podem apontar caminhos promissores de intervençóes centradas no fortalecimento das capacidades do indivíduo e consequente prevenção de comportamentos negativos, como ansiedade, depressão, baixa autoestima e comportamento autodestrutivo. Ademais, podem guiar a universidade no desenvolvimento de iniciativas abrangentes e efetivas de atenção à saúde mental de futuros profissionais.

Investigações sobre a autoeficácia são escassas, sobretudo comparando-a com outras condiçôes relacionadas à saúde mental e em cenário brasileiro. No contexto da aprendizagem, a autoeficácia é geralmente o aspecto que se busca fortalecer, para assim, formar pessoas competentes, seguras, determinadas, motivadas, confiantes e que persistam perante adversidades.

Assim, diante dessa lacuna do conhecimento, o presente estudo teve como objetivo identificar a relação da autoeficácia percebida com a autoestima e a presença dos sintomas de ansiedade e depressão de estudantes no início do curso de graduação em enfermagem. A hipótese é de que os participantes com sintomas ansiosos e depressivos e reduzida autoestima possuam baixa percepção de eficácia pessoal.

\section{Métodos}

Estudo correlacional e transversal, desenvolvido em duas Instituiçóes de Ensino Superior (IES) públicas, que oferecem ensino, pesquisa e extensão a alunos do curso de enfermagem e outros, em regime integral. A escolha dessas instituiçóes ocorreu pela inserçáo dos pesquisadores nos campos de coletas de dados, bem como pela oportunidade de verificar possíveis diferenças na saúde mental dos participantes de diferentes regióes do Brasil (Sudeste e Centro-Oeste).

A população foi constituída por todos os alunos matriculados no $1^{\circ}$ ano de graduação em enfermagem das IES $1(n=60)$ e $2(n=30)$. A preferência pelo ingressante se justificou por representar um período de transição de ciclos, mudança de ambiente e rotinas, além de maior demanda por responsabilidades e autonomia.

Foram estabelecidos como critérios de elegibilidade: estar matriculado no $1^{\circ}$ ano do curso, não possuir histórico de reprovação, ter 18 anos ou mais e estar presente nas datas de coleta de dados. Por se tratar de estudo de base populacional, não se realizou cálculo de amostra. Dos 90 possíveis participantes, excluíram-se dois em decorrência de reprovação e seis por ausência na coleta de dados. Desta forma, a amostra foi constituída por 82 estudantes, cuja representatividade foi de $91,1 \%$ da população.

Para caraterização dos participantes foi aplicado um questionário constituído por variáveis pessoais (sexo, idade, estado civil, procedência); acadêmicas (satisfação com o curso e profissão, se a enfermagem foi sua primeira opção no vestibular, ideação de abandono do curso); sociais (sustento na universidade, exercício de atividades profissionais remuneradas e sobrecarga com as atividades acadêmicas).

Para avaliação da saúde mental foram empregados os seguintes instrumentos: Escala de Autoeficácia Geral e Percebida, Escala de Autoestima Rosenberg (EAR) e Escala Hospitalar de Ansiedade e Depressão HADS.

A Escala de Autoeficácia Geral e Percebida é composta por 10 itens, com respostas que variam de um a cinco. Cada item mensura o alcance de metas e à percepção interna de sucesso. Maior pontuação indica maior percepção da autoeficácia, em um intervalo de 10 a 50. ${ }^{(11)}$ Essa escala, adaptada e validada para diversos países, apresentou boa consistência interna e adequação para uso no Brasil, com alfa de Cronbach $(\alpha)$ de 0,81 . $^{(11)}$

A EAR, validada para o português em 2001, ${ }^{(12)}$ tem sido a escala mais empregada na literatura nacional e internacional para avaliar a autoestima, com elevada consistência interna $(\alpha=0,90) .{ }^{(13)}$ É apresentada na forma Likert, de quatro pontos, variando entre "concordo plenamente" a "discordo plenamente". É composta por 10 itens que mensuram uma única dimensão: cinco para avaliação de sentimentos positivos do indivíduo sobre si mesmo e cinco de sentimentos negativos. A somatória das respostas pode variar de 10 a 40 pontos, sendo a au- 
toestima interpretada da seguinte forma: $>30$ pontos=alta ou satisfatória; de 20 a 30 pontos=média; $<20$ pontos=baixa ou insatisfatória. ${ }^{(12)}$

A HADS possuía proposta inicial de mensurar sintomas sugestivos de ansiedade e depressão em contexto clínico hospitalar, mas posteriormente passou a ser empregada em outros ambientes, incluindo pessoas sadias e estudantes do ensino superior. ${ }^{(14,15)}$ Essa escala tem a função de rastrear sintomas e não de estabelecer diagnósticos. Ademais, é amplamente empregada na literatura por ser de fácil compreensão, objetiva e consistente (HADS-A $\alpha=0,79$ e HADS-D $\alpha=0,84) .{ }^{(16)}$ Possui 14 questóes intercaladas de ansiedade e depressão. Cada item da HADS contempla quatro possibilidades de respostas ( 0 a 3 ), totalizando pontuação máxima de 21 pontos para cada subescala. Estudantes com escore igual ou maior a nove em cada subescala foram considerados com sintomatologia de ansiedade e/ou depressão presentes. ${ }^{(16)}$

Os estudantes foram identificados por meio de lista de presença oferecida pelos coordenadores de curso das IES 1 e 2 . A abordagem aos universitários foi iniciada de forma presencial, em sala de aula, com um convite para a participação na pesquisa. Após o aceite e assinatura do TCLE, foi iniciada a coleta dos dados, em setembro de 2018, pelos próprios pesquisadores.

Para garantir a equivalência na coleta dos dados nas duas IES, os pesquisadores uniformizaram a forma de aplicação dos instrumentos. Estes foram distribuídos a cada aluno em sua carteira de estudo, sem a influência dos pesquisadores ou professores, em um tempo médio de 30 minutos. Em seguida, iniciou-se a leitura das instruçóes de preenchimento dos instrumentos e, ao final, os alunos depositavam suas respostas em envelope fechado.

Os dados foram processados e analisados por meio do programa Minitab 17 (Minitab Inc.). Foram realizadas análises descritivas para as variáveis de caracterização amostral, como sexo, estado civil, procedência, satisfação com o curso, dentre outros.

O teste de Análise de Variância com teste de comparação múltipla de Games-Howell foi utilizado para a comparação dos escores de percepção de autoeficácia com as variáveis categóricas com mais de dois níveis. $\mathrm{O}$ teste $\mathrm{t}$ para amostras independen- tes foi empregado para a comparação dos escores de autoeficácia com as variáveis categóricas com até 2 níveis e o teste de correlação de Spearman para analisar os escores de autoeficácia e a idade dos estudantes. A normalidade dos dados foi verificada pelo teste de Anderson-Darling, e o nível de significância aplicado para os testes foi de 0,05 ou $5 \%$.

Esse estudo foi aprovado pelos Comitês de Ética em Pesquisa, sob o número 2.691.383, em junho de 2018 (IES 1) e sob o número 2.920.649, em setembro de 2018 (IES 2) e atende à resolução n. ${ }^{\circ}$ 466/12, , $^{(17)}$ referente às pesquisas que envolvem seres humanos.

\section{Resultados}

Participaram desse estudo 82 estudantes do $1^{\circ}$ ano da graduação em enfermagem. A maioria era do sexo feminino $(71 ; 86,6 \%)$, não possuía companheiro $(76 ; 92,7 \%)$ e pertencia a IES $1(52 ; 63,4 \%)$. A maior parte dos estudantes relatou morar com os pais $(39 ; 47,6 \%)$, ter relação familiar harmoniosa (70; 85,4\%), possuir recursos financeiros suficientes para seu sustento $(50 ; 61,0 \%)$ e náo exercer trabalho remunerado (76; 92,7\%).

Parcela representativa dos participantes afirmou que a enfermagem não foi sua primeira opção de curso no vestibular $(48 ; 59,3 \%)$, sendo que destes 48 estudantes, $36(73,5 \%)$ desejariam ter cursado medicina. A maioria se diz satisfeita com o curso (76; $92,7 \%)$, com a profissão $(76 ; 92,7 \%)$ e não pensa em abandoná-la (49; 59,8\%), mas se sente sobrecarregada com as atividades que a envolvem (70; 85,4\%).

A média da idade dos 82 estudantes avaliados foi de 19,8 anos, com desvio padrão de 3,9 anos e mediana de 19,0 anos. O coeficiente de variação dessa distribuição foi de 19,8\%. A idade mínima observada foi de 18,0 anos e a máxima de 39,0 anos.

A maioria dos estudantes avaliados apresentou sintomas ansiosos $(60 ; 73,2 \%)$ e autoestima moderada (73; 89,0\%). Apenas um participante foi classificado com autoestima alta $(1,2 \%)$ e os sintomas depressivos estiveram presentes em $31,7 \%$ da amostra $(n=26)$.

A percepção de autoeficácia dos estudantes foi moderada, com média de 31,4 pontos (variação da escala 10 a 50 pontos), desvio padrão de 7,4 pontos 
e mediana de 32,0 pontos. $\mathrm{O}$ coeficiente de variação da distribuição foi 23,7\%, valor mínimo de 15,0 pontos e máximo de 50,0 pontos. Não houve diferença estatística nos escores de percepção da autoeficácia entre os participantes das IES 1 e $2(\mathrm{P}=0,453)$

Foi possível observar relação entre a percepção de autoeficácia e os sintomas ansiosos (HADS-A; $\mathrm{P}<0,001)$ e depressivos (HADS-D; $\mathrm{P}<0,001$ ). Estudantes com ansiedade e depressão apresentaram escores de autoeficácia percebida inferiores àqueles sem ansiedade e sem depressão. As demais variáveis categóricas não foram associadas a autoeficácia dos participantes $(\mathrm{p}>0,05)$ (Tabela 1$)$.

Tabela 1. Relação entre a percepção de autoeficácia e as variáveis de caracterização amostral, autoestima, sintomas ansiosos e depressivos $(\mathrm{n}=82)$

\begin{tabular}{|c|c|c|c|}
\hline \multirow[b]{2}{*}{ Variáveis categóricas } & \multicolumn{2}{|c|}{ Percepção de autoeficácia } & \multirow[b]{2}{*}{$p$-value ${ }^{*}$} \\
\hline & $n(\%)$ & $\begin{array}{l}\text { Média } \pm \text { desvio } \\
\text { padrão }\end{array}$ & \\
\hline \multicolumn{4}{|l|}{ Sexo } \\
\hline Feminino & $71(86,6)$ & $31,0 \pm 7,4$ & 0,238 \\
\hline Masculino & $11(13,4)$ & $34,1 \pm 7,6$ & \\
\hline \multicolumn{4}{|l|}{ Estado civil } \\
\hline Com companheiro & $6(7,3)$ & $33,5 \pm 7,1$ & 0,496 \\
\hline Sem companheiro & $76(92,7)$ & $31,3 \pm 7,5$ & \\
\hline \multicolumn{4}{|c|}{ Instituição de Ensino - IES } \\
\hline IES1 & $52(63,4)$ & $31,9 \pm 7,1$ & 0,453 \\
\hline IES2 & $30(36,6)$ & $30,6 \pm 8,1$ & \\
\hline \multicolumn{4}{|l|}{ Com quem reside } \\
\hline Pais & $39(47,6)$ & $32,1 \pm 6,9$ & $0,823^{* *}$ \\
\hline Avós & $2(2,4)$ & $32,0 \pm 12,7$ & \\
\hline Outros & $41(50,0)$ & $30,8 \pm 7,9$ & \\
\hline \multicolumn{4}{|l|}{ Relação familiar } \\
\hline Conflituosa & $12(14,6)$ & $33,4 \pm 6,1$ & 0,259 \\
\hline Harmoniosa & $70(85,4)$ & $31,1 \pm 7,7$ & \\
\hline \multicolumn{4}{|l|}{ Satisfeito com a profissão } \\
\hline Não & $6(7,3)$ & $30,3 \pm 8,7$ & 0,755 \\
\hline $\operatorname{Sim}$ & $76(92,7)$ & $31,5 \pm 7,4$ & \\
\hline \multicolumn{4}{|l|}{ Satisfeito com o curso } \\
\hline Não & $6(7,3)$ & $28,3 \pm 9,1$ & 0,419 \\
\hline Sim & $76(92,7)$ & $31,7 \pm 7,3$ & \\
\hline \multicolumn{4}{|l|}{ Sente-se sobrecarregado } \\
\hline Não & $12(14,6)$ & $34,0 \pm 8,2$ & 0,255 \\
\hline $\operatorname{Sim}$ & $70(85,4)$ & $31,0 \pm 7,3$ & \\
\hline \multicolumn{4}{|l|}{ Autoestima $^{3}$} \\
\hline Baixa & $8(9,8)$ & $30,1 \pm 6,2$ & 0,551 \\
\hline Moderada e alta & $74(90,2)$ & $31,6 \pm 7,6$ & \\
\hline \multicolumn{4}{|l|}{ HADS-A } \\
\hline Com ansiedade & $60(73,2)$ & $29,4 \pm 6,9$ & $<0,001$ \\
\hline Sem ansiedade & $22(26,8)$ & $37,1 \pm 5,7$ & \\
\hline \multicolumn{4}{|l|}{ HADS-D } \\
\hline Com depressão & $26(31,7)$ & $26,5 \pm 6,9$ & $<0,001$ \\
\hline Sem depressão & $56(68,3)$ & $33,7 \pm 6,6$ & \\
\hline
\end{tabular}

" $p$-value referente ao teste t para amostras independentes a $\mathrm{P}<0,05$; "Valor $\mathrm{P}$ referente ao teste de Análise de Variância (ANOVA) a $\mathrm{P}<0,05$; ${ }^{3} \mathrm{Um}$ indivíduo da distribuição com classificação de autoestima Rosenberg ALTA foi agrupado com os demais indivíduos com autoestima Rosenberg MODERADA para tornar possivel a análise associativa
Os resultados também demonstraram correlação muito fraca entre os escores de autoeficácia percebida e a idade dos estudantes ( $\mathrm{r}=0,197 ; \mathrm{P}=0,076)$.

\section{Discussão}

Ainda que essa investigação, cujo objetivo foi verificar a relação da autoeficácia com a autoestima e os sintomas ansiosos e depressivos, tenha sido conduzida em dois centros e com amostra representativa, algumas limitaçóes devem ser consideradas. Uma delas se refere ao delineamento transversal, que impede a avaliação das variáveis ao longo do tempo. Destaca-se, ainda, a não mensuração de variáveis que poderiam ter influência na percepção de autoeficácia dos participantes, como: estresse, dinâmica familiar, formas de enfrentamento, apoio social e vícios. Desta forma, outras investigações são necessárias para clarificar essas lacunas.

No entanto, os resultados da presente pesquisa agregam dados científicos relevantes acerca da saúde mental dos universitários que cursam enfermagem e sobre alguns indicadores de bem-estar que podem ser fortalecidos para evitar o adoecimento dos graduandos. A autoeficácia tem se mostrado um determinante promissor na edificação de recursos psíquicos, uma vez que prepara o jovem para o enfrentamento assertivo do estresse pessoal e acadêmico, além de amortecer os efeitos negativos de depressão e ansiedade. ${ }^{(18)}$

Dessa forma, os presentes dados refletem um avanço no contexto da prevenção primária, pois demonstra a importância de um olhar ampliado a esses recursos emocionais positivos em jovens, sobretudo nos primeiros anos da graduação. Essas estratégias são essenciais para prevenção da instalação de condiçóes clínicas e mentais negativas, em detrimento de programas voltados para estudantes com quadros de depressão e ansiedade já instalados.

A partir desses fatores, esse estudo abre caminhos para o desenvolvimento de programas psicoeducacionais dentro da universidade, que possam estimular e ensinar os estudantes a fortalecer suas competências emocionais, com foco na promoção da autoeficácia, da autoestima, das habilidades nos inter-relaciona- 
mentos e na utilização de estratégias de enfrentamento positivas. Essas medidas, em médio e longo prazo, poderão propiciar enfermeiros mais saudáveis, satisfeitos e que proporcionarão a seus clientes uma assistência competente e segura. ${ }^{(19)}$

Os resultados dessa pesquisa revelaram autoeficácia moderada entre os participantes e a relação inversa desse constructo com os sintomas de ansiedade e depressão.

Recentes evidências científicas corroboram esses $\operatorname{achados}^{(19,20)}$ e esclarecem que os níveis satisfatórios de eficácia pessoal desempenham importante papel na proteção dos transtornos de humor e ansiedade ${ }^{(18,21,22)}$ já que podem atuar como um recurso psicológico na superação do estresse. ${ }^{(18,21)}$ Ao investigar o papel da autoeficácia na saúde mental positiva e negativa de estudantes alemães, os resultados sugeriram que ela funciona como um amortecedor do estresse diário, conferindo efeito protetor à saúde mental desses indivíduos. ${ }^{(21)}$

Apoiando esses resultados, dados de 2160 universitários chineses, avaliados ao longo de um ano, mostraram que estressores diários estavam associados ao aumento dos sintomas de depressão, ansiedade e estresse e à diminuição do bem-estar. A autoeficácia foi apontada como mediadora entre o estresse e essas condiçóes mentais, ao mesmo tempo em que foi diretamente relacionada à saúde mental positiva dos participantes. ${ }^{(18)}$ Postula-se que diante de uma situação desafiadora, indivíduos com boa eficácia pessoal são mais confiantes em sua capacidade de enfrentar os desafios e experimentam melhor satisfação com a vida e bem-estar. ${ }^{(22)}$

Pesquisa clínica conduzida em três universidades da Ásia concluiu que a elevada autoeficácia contribuiu para a melhora da depressão e atribuiu esse resultado ao maior reconhecimento, por parte dos alunos, de seus pontos fortes e do uso dessa ferramenta de formas inéditas e diferentes. Aponta que os participantes foram capazes de se dedicar aos estudos de forma mais eficaz e de lidar com as tarefas acadêmicas com melhor habilidade. Ademais, foram observados maiores índices de sensação de sucesso, empoderamento e emoções positivas. ${ }^{(19)}$

Postula-se que os resultados dos estudos descritos apoiam um corpo de evidências que fundamen- tam a relação inversa entre a percepção de autoeficácia com ansiedade e depressão encontrada nesse estudo.

Nessa pesquisa, a autoeficácia não teve relação com as demais características sociodemográficas, entretanto, é possível identificar na literatura estudos apontando certas tendências, como a associação com a relação familiar e a autoestima. ${ }^{(23,24)}$

A relação familiar conflituosa foi apontada como associada à autoeficácia geral diminuída, em adolescentes. No entanto, demonstrou-se que mesmo em situações de conflitos familiares, adolescentes que encontraram apoio social na interação com seus pares e sucesso acadêmico tinham seus níveis de autoeficácia aumentados, compensando os efeitos das relaçóes parentais negativas. ${ }^{(23)}$ Esse resultado evidencia o importante papel da universidade na construção do indivíduo, da competência profissional e de redes de apoio que amparem e amorteçam os efeitos negativos de possíveis relaçóes conflituosas. Acrescenta-se que a mensuraçáo dessa informação em nossa pesquisa foi objetiva e dicotômica e náo de forma detalhada, com o uso de instrumentos específicos, como no estudo que identificou tal relação ${ }^{(24)}$ e talvez, por isso, náo tenhamos encontrado relação significativa.

Divergindo dos atuais achados, a relaçáo positiva entre a autoeficácia e autoestima têm sido confirmada em estudos envolvendo universitários. ${ }^{(19,25-27)}$ As evidências recaem na edificação da saúde mental positiva. Em outras palavras, a confiança do indivíduo em sua própria capacidade contribui para manutenção de ótimos níveis de autoestima, uma vez que os indivíduos sentem que têm maior capacidade de enfrentar situaçôes estressantes. ${ }^{27)}$

Adicionalmente, a literatura destaca que o ambiente acadêmico, as relaçóes estabelecidas com autoridades educacionais, como professores e enfermeiros clínicos, ao longo da graduação, também podem influenciar os níveis de autoeficácia de estudantes de enfermagem. Interaçóes pautadas no acolhimento, cooperação, respeito e confiança são capazes de influenciar positivamente esse constructo nesses indivíduos. ${ }^{(28)}$ Mediante estes resultados, é recomendável que as IES, por meio de programas preventivos, auxiliem o desenvolvimento emocional e acadêmico de 
seus alunos, com açóes de promoção a saúde mental, identificação precoce de emoçôes negativas e oferecimento de apoio psicológico aos estudantes vulneráveis, ${ }^{(3)}$ sobretudo àqueles no início do curso.

Retratando e reforçando a importância dos aspectos mentais aqui estudados, não só durante o percurso acadêmico, mas também como ferramentas imprescindíveis para atuação profissional, um recente estudo realizado com 1307 enfermeiras, com idades compreendidas entre 22 e 60 anos, demonstrou que profissionais com altos níveis de autoeficácia e de autoestima obtiveram menores pontuaçóes na escala de Burnout. Estes achados confirmam as implicaçôes benéficas das crenças e atitudes positivas do indivíduo em relação a si mesmo sobre os efeitos negativos da carga de trabalho. Ademais, os profissionais com altos níveis de eficácia pessoal também obtiveram maior pontuação na autoestima global, refletindo a íntima relação destas variáveis. ${ }^{(29)}$

\section{Conclusão}

Os achados deste estudo indicaram autoestima e percepção de autoeficácia moderadas, além de sintomas elevados de ansiedade e depressão. Escores reduzidos de autoeficácia percebida foram relacionados à presença de sintomas ansiosos e depressivos. Esses resultados refletem o comprometimento do bem-estar dos alunos e a necessidade de cuidados efetivos e atrativos à saúde mental no âmbito acadêmico, especialmente nos primeiros anos da graduação em enfermagem.

\section{Agradecimentos}

O presente trabalho foi realizado com apoio da Fundação de Amparo à Pesquisa do Estado de São Paulo (FAPESP), processo no 2018/14743-9.

\section{Colaborações}

Melo HE, Severian PFG, Eid LP, Souza MR, Sequeira CAC, Souza MGG e Pompeo DA contri- buíram com a concepção do projeto, análise e interpretação dos dados, redação do artigo, revisão crítica relevante do conteúdo intelectual e aprovação final da versão a ser publicada.

\section{Referências}

1. Karaca A, Yildirim N, Cangur S, Acikgoz F, Akkus D. Relationship between mental health of nursing students and coping, self-esteem and social support. Nurse Educ Today. 2019 May;76:44-50.

2. Tung YJ, Lo KK, Ho RC, Tam WS. Prevalence of depression among nursing students: A systematic review and meta-analysis. Nurse Educ Today. $2018 ; 63: 119-29$.

3. Abu Ruz ME, Al-Akash HY, Jarrah S. Persistent (anxiety and depression) affected academic achievement and absenteeism in nursing students. Open Nurs J. 2018;12(1):171-9.

4. Sakellari E, Psychogiou M, Georgiou A, Papanidi M, Vlachou V, Sapountzi-Krepia D. Exploring religiosity, self-esteem, stress, and depression among students of a Cypriot University. J Relig Health. 2018;57(1):136-45.

5. Fernandes MA, Vieira FE, Silva JS, Avelino FV, Santos JD. Prevalence of anxious and depressive symptoms in college students of a public institution. Rev Bras Enferm. 2018;71 Suppl 5:2169-75.

6. Montes-Hidalgo J, Tomás-Sábado J. Self-esteem, resilience, locus of control and suicide risk in nursing students. Enferm Clin. 2016;26(3):188-93.

7. Bandura A. Social cognitive theory: an agentic perspective. Annu Rev Psychol. 2001;52(1):1-26.

8. Masoudi Alavi N. Self-efficacy in nursing students. Nurs Midwifery Stud. 2014;3(4):e25881.

9. Valizadeh L, Zamanzadeh V, Gargari RB, Ghahramanian A, Tabrizi FJ, Keogh B. Pressure and protective factors influencing nursing students' self-esteem: A content analysis study. Nurse Educ Today. 2016;36:468-72.

10. Li C, Yin H, Zhao J, Shang B, Hu M, Zhang P, et al. Interventions to promote mental health in nursing students: A systematic review and meta-analysis of randomized controlled trials. J Adv Nurs. 2018;74(12):2727-41.

11. Souza I, Souza MA. Validação da escala de autoeficácia geral percebida. Rev Univ Rural. 2004;26(1-2):12-7.

12. Dini GM, Quaresma MR, Ferreira LM. Translation into portuguese, cultural adaptation and validation of the Rosenberg Self-esteem Scale. Rev Bras Cir Plást. 2004;19(1):41-52.

13. Hutz CS, Zanon C. Revisão da apadtação, validação e normatização da escala de autoestima de Rosenberg. Aval Psicol. 2011; 10 (1) : 41-4.

14. Francis B, Gill JS, Yit Han N, Petrus CF, Azhar FL, Ahmad Sabki Z, et al. Religious coping, religiosity, depression and anxiety among medical students in a multi-religious setting. Int J Environ Res Public Health. 2019;16(2):259.

15. Spadaro KC, Hunker DF. Exploring the effects of an online asynchronous mindfulness meditation intervention with nursing students on stress, mood, and cognition: a descriptive study. Nurse Educ Today. 2016;39:163-9. 
16. Marcolino JA, Mathias LA, Piccinini Filho L, Guaratini AA, Suzuki FM, Alli LA. Hospital Anxiety and Depression Scale: a study on the validation of the criteria and reliability on preoperative patients. Rev Bras Anestesiol. 2007;57(1):52-62.

17. Brasil. Ministério da Saúde. Conselho Nacional de Saúde. Resolução n. 466, de 12 de dezembro de 2012. Aprova diretrizes e normas regulamentadoras de pesquisas envolvendo seres humanos. Brasília (DF), Diário Oficial da União, 12 dez. 2012.

18. Schönfeld P, Brailovskaia J, Zhang XC, Margraf J. Self-efficacy as a mechanism linking daily stress to mental health in students: a three-wave cross-lagged study. Psychol Rep. 2019;122(6):207495.

19. Guo YF, Zhang X, Plummer V, Lam L, Cross W, Zhang JP. Positive psychotherapy for depression and self-efficacy in undergraduate nursing students: A randomized, controlled trial. Int J Ment Health Nurs. 2017 Aug;26(4):375-83.

20. Abdal M, Masoudi Alavi N, Adib-Hajbaghery M. Clinical self-efficacy in senior nursing students: a mixed- methods study. Nurs Midwifery Stud. 2015;4(3):e29143.

21. Schönfeld P, Brailovskaia J, Bieda A, Zhang XC, Margraf J. The effects of daily stress on positive and negative mental health: mediation through self-efficacy. Int J Clin Health Psychol. 2016;16(1):1-10.

22. Lee J, Kim E, Wachholtz A. The effect of perceived stress on life satisfaction : the mediating effect of self-efficacy. Chongsonyonhak Yongu. 2016;23(10):29-47.
23. McCauley DM, Weymouth BB, Feinberg ME, Fosco GM. Evaluating school and peer protective factors in the effects of interparental conflict on adolescent threat appraisals and self-efficacy. J Adolesc. 2019;71:28-37.

24. Fosco GM, Feinberg ME. Cascading effects of interparental conflict in adolescence: linking threat appraisals, self-efficacy, and adjustment. Dev Psychopathol. 2015;27(1):239-52.

25. Terp U, Hjärthag F, Bisholt B. Effects of a cognitive behavioral-based stress management Program on Stress Management Competency, Self-efficacy and Self-esteem experienced by nursing students. Nurse Educ. 2019;44(1):E1-5.

26. Ribeiro RM, Bragiola JV, Eid LP, Ribeiro RC, Sequeira CA, Pompeo DA. Impacto de uma intervenção através do Facebook para fortalecimento da autoestima em estudantes de enfermagem. Rev Latino Am Enfermagem. 2020;28:e3237.

27. Pérez-Fuentes MD, Jurado MD, Gázquez Linares JJ. Explanatory value of general self-efficacy, empathy and emotional intelligence in overall self-esteem of healthcare professionals. Soc Work Public Health. 2019;34(4):318-29.

28. Sohrabi Z, Kheirkhah M, Sahebzad ES, Rasoulighasemlouei S, Khavandi S. Correlation between Students' self-efficacy and teachers' educational leadership style in Iranian midwifery students. Glob J Health Sci. 2015;8(7):260-5.

29. Molero MD, Pérez-Fuentes MD, Gázquez JJ. Analysis of the mediating role of self-efficacy and self-esteem on the effect of workload on burnout's influence on nurses' plans to work longer. Front Psychol. 2018;9:2605. 